\title{
Voter Choice in Multi-Party Democracies: A Test of Competing Theories and Models
}

\author{
Kevin M. Quinn, Harvard University \\ Andrew D. Martin, State University of New York at Stony Brook \\ Andrew B. Whitford, Rice University
}

We contrast social-structural theories of voting behavior with spatial theories of voting behavior to explain voter choice in the Netherlands and Great Britain. We hypothesize that voting behavior is best explained by the spatial theory of voting. Markov chain Monte Carlo (MCMC) simulation is used to estimate multinomial probit (MNP) and multinomial logit (MNL) models of voter choice, for which we calculate Bayes factors for the purpose of model comparison. We find that the joint social-structural/spatial model is the best explanatory model in the Netherlands. Our results indicate that the MNP model outperforms the MNL model in our Dutch sample. In Great Britain, on the other hand, a purely spatial model is the best explanatory model, and our MNL model outperforms our MNP model. These results suggest the question of whether to employ MNL or MNP depends crucially on the data at hand.

\section{INTRODUCTION}

Over the past forty years, researchers have accumulated a great deal of knowledge about the determinants of voter choice in what are essentially two-party systems. Yet, our empirical understanding of how voters choose candidates in multi-party systems has not developed to the same degree. This lack of understanding does not stem from a dearth of competing explanations. Instead, as Whitten and Palmer (1996) suggest, it is in part the result of methodological problems associated with modeling multiple, unordered alternative choices. Since a large share of empirical work in multi-party settings uses statistical models that misrepresent the causal processes implied by competing explanations, it is not clear what such results tell us about the fit of various theories of multi-party vote choice. Even when researchers

An earlier version of this paper was presented at the Annual Meeting of the American Political Science Association, San Francisco, California, August 29-September 1, 1996. We thank Michael Alvarez, Sid Chib, Ed Greenberg, Jonathan Katz, Eric Lawrence, Lanny Martin, Jonathan Nagler, Norman Schofield, Itai Sened, and three anonymous reviewers for helpful comments and discussions. Special thanks go to Sid Chib, Ed Greenberg, and Yuxin Chen for allowing us to use their multinomial probit code. This work is based on research supported under National Science Foundation Grant SBR 96-17708. Additional financial support was provided by The Center in Political Economy at Washington University. All data to replicate this study, as well as tables and figures omitted because of space limitations, are available at http://psweb.sbs.ohio-state.edu/ajps/. Responsibility for all remaining errors lies solely with the authors.

American Journal of Political Science, Vol. 43, No. 4, October 1999, Pp. 1231-1247 @1999 by the Midwest Political Science Association 
employ a statistical model that could plausibly have generated the observed data, they generally do not compare the fit of this model to other plausible models.

Here, we test two competing explanations of voting behavior in the Netherlands with special emphasis on obtaining both a reasonable functional form as well as the proper model specification. We evaluate explanations based on social-structural and spatial theories of voting. We use statistical techniques that both accurately represent the underlying causal models and allow us to determine the proper model specification and the correct functional form. By employing a Bayesian approach, we are able to compare both explanations and models on the scale of probability to determine whether the (oftentimes substantial) additional model complexity and computational difficulties of the multinomial probit (MNP) model over the simpler multinomial logit (MNL) model will always buy more explanatory power. We then extend our analysis by comparing the same theories and models in Great Britain. We conclude that it is inappropriate to assume that MNP is globally superior to MNL in all contexts.

\section{Voting Behavior in The NetherLands}

The Netherlands is a critical case for the comparison of competing explanations of voting behavior in multi-party democracies for a number of reasons: a substantial body of empirical analysis addresses voting behavior in the Netherlands (e.g., Andeweg and Irwin 1993); previous studies of Dutch voting behavior indicate varying degrees of support for each of the explanations; and because Dutch legislators are elected via national-list proportional representation, district-specific factors are constant across the population, and we can safely ignore them in our statistical models.

Prior to the late 1960s, social-structural explanations of voter behavior dominated the literature. Scholars characterized the Dutch electoral system by verzuiling, or pillarisation (e.g., Lijphart 1975). As Rose and McAllister note:

The Netherlands has been a classic example of a structured system of multiparty competition, because the electorate has been determined along two dimensions, religion and class, each sustaining separate political parties.... When the electorate is determined, voting reflects the persisting structure of society. Individual votes are not cast according to the voluntaristic choice of individuals. ... (1986, 12, 8; cited in Andeweg and Irwin 1993, 97; emphasis added).

Those professing religious beliefs voted for their denomination's religious party; members of the working class without strong religious beliefs voted for Labor; and the nonreligious middle class voted for Liberal. 
Another common explanation, the spatial model, contends that Dutch voters care about the policies advocated by the parties and vote accordingly (Dutter 1985; Downs 1957; Enelow and Hinich 1984). The spatial model assumes that government policy can be summarized by a point in a well-defined issue space, that voter utility functions are defined over this space, and that voters vote for the party that puts forth the policy proposal that provides them with the highest utility.

Even though the social-structural and spatial explanations have received some empirical support, few have empirically assessed the relative importance of these explanations in Western Europe. ${ }^{1}$ Furthermore, the choice to employ either MNL or MNP has generally not been based on rigorous model selection criteria. In some applications (e.g., Lacy and Burden 1999) there are strong theoretical reasons for choosing one model over the other, but this is generally not true. Moreover, Alvarez and Nagler (1998) demonstrate that in some cases MNL and MNP lead to different substantive inferences. Nonetheless, because they are nonnested, there is no simple way directly to compare the fit of these competing models using tests based on classical inference.

\section{Models of Multinomial Response}

In this section, we consider the properties of MNL and MNP and show how to use the techniques of Bayesian inference to choose between them. The choice between multinomial response models is not always simple; the greater flexibility of the MNP model might seem to make it preferable to the more restrictive MNL, but as we show below, this is not always true. Bayesian inference provides a consistent and computationally practical means to choose between nonnested models with different functional forms.

\subsection{Random Utility Motivation}

Given data from $n$ voters choosing between $p$ alternatives (parties), both MNP and MNL can be motivated by the following random utility model:

$$
\mathbf{z}_{i}=\mathbf{V}_{i} \gamma+\mathbf{W}_{i} \delta+\mathbf{u}_{i}
$$

${ }^{1}$ But see Whitten and Palmer (1996) and Alvarez and Nagler (1998). They do assess the relative impact of issue positions and ideology vs. the impact of sociological variables on voting behavior, but take the matter only so far. For example, Whitten and Palmer consider only voters' issue preferences rather than the difference between voters' preferences and party issue positions. Alvarez and Nagler measure ideological distance as the difference between voters' ideological self-placement and the mean perceived party locations. Given the multidimensional nature of the Dutch issue space, the approach we adopt provides a more complete and substantively meaningful account of issue voting in the Netherlands (see also Schofield et al. 1998). 


$$
y_{i j}=\left\{\begin{array}{l}
1 \text { if } z_{i j}=\max \left(\mathbf{z}_{\mathbf{i}}\right) \\
0 \text { otherwise }
\end{array} \quad \text { for } i=1, \ldots, n \text { and } j=1, \ldots, p\right.
$$

where $\mathbf{z}_{\mathrm{i}}$ is a $p \times 1$ vector, with $z_{i j}$ defined as the utility voter $i$ attaches to voting for party $j$; $\mathbf{V}_{\mathrm{i}}$ is a $p \times 1$ matrix of choice-specific covariates; $\mathbf{W}_{\mathrm{i}}$ is a $p \times m$ matrix of individual specific covariates ${ }^{2} \gamma$ and $\delta$ are vectors of choice-specific and individual-specific coefficients, respectively; $\mathbf{u}_{\mathrm{i}}$ is a $p \times 1$ vector of disturbances; and $\mathbf{y}_{\mathrm{i}}$ is a $p \times 1$ vector representing the observed vote choice of individual $i$. The probability that alternative $j$ is chosen by individual $i$ is simply the probability that $z_{i j}$ is equal to $\max \left(\mathbf{z}_{\mathrm{i}}\right)$. MNP and MNL differ in how the disturbance terms are assumed to be distributed.

\subsection{The MNP Model}

The multinomial probit model results from the assumption that the errors in Equation 1 are distributed multivariate normal with mean vector $\mathbf{0}$ and variance-covariance matrix $\boldsymbol{\Omega}$. To identify the MNP, Equation 1 has to be slightly reformulated. One identification problem is that an arbitrary constant can be added to both sides of Equation 1 without changing the distribution of $\mathbf{y}_{\mathrm{i}}$. To remedy this problem, it is customary to express each $z_{i j}$ relative to $z_{i p}$. Define $\mathbf{z}_{i}^{*}=\left(z_{i 1}^{*}, \ldots, z_{i, p-1}^{*}\right)$ where $z_{i j}^{*}=z_{i j}-z_{i p}$. The underlying regression model is now $\mathbf{z}_{i}^{*}=\mathbf{X}_{i} \boldsymbol{\beta}+\varepsilon_{i}$ where $\varepsilon_{i} \sim N_{p-1}(\mathbf{O}, \mathbf{\Sigma})$ is a $(p-1) \times 1$ vector of errors, and $\mathbf{X}_{\mathrm{i}}$ is the new matrix of covariates obtained by horizontally concatenating $\mathbf{V}_{i}^{*}=\mathbf{V}_{i}-\mathbf{v}_{i p}^{\prime}$ to $\mathbf{W}_{\mathrm{i}}$ and then deleting the $p$ th row of $\mathbf{X}$ and each column of individual-specific attributes for the $p$ th choice category. ${ }^{3}$ A second identification problem is that multiplying $\mathbf{z}_{i}^{*}$ by a positive constant will not change the value of $\mathbf{y}_{i}$. This problem is traditionally solved by restricting $\boldsymbol{\Sigma}_{11}$ to be equal to 1 . For notational purposes, we refer to the restricted matrix as $\Sigma^{*}$.

To form the MNP sampling density, begin by noting that the probability that individual $i$ chooses party $j$ is

$$
\operatorname{Pr}\left(y_{i j}=1 \mid \boldsymbol{\beta}, \mathbf{\Sigma}^{*}\right)=\int_{A_{j}} \phi_{p-1}\left(\mathbf{z}_{i}^{*} \mid \mathbf{X}_{i} \boldsymbol{\beta}, \mathbf{\Sigma}^{*}\right) d \mathbf{z}_{i}^{*}
$$

\footnotetext{
${ }^{2}$ We assume that $\mathbf{W}$ is formed as $\mathbf{W}^{*} \otimes \mathbf{I}_{p}$ where $\mathbf{W}^{*}$ is the original $n \times m^{*}$ matrix of $m^{*}$ individual-specific attributes from all $n$ individuals.

${ }^{3}$ Note that if $\boldsymbol{\Omega}=\mathbf{I}_{\mathbf{p}}$, then $\mathbf{\Sigma}=\mathbf{I}_{\mathbf{p}-\mathbf{1}}+\mathbf{1 1}^{\prime}$. This can be normalized to $\mathbf{\Sigma}=\frac{1}{2} \cdot \mathbf{I}_{\mathbf{p}-\mathbf{1}}+\frac{1}{2} \cdot \mathbf{1 1}^{\prime}$. This follows directly from the rules for calculating the variance and covariance of sums and differences of random variables (see DeGroot 1986, p. 216). Not only will $\mathbf{\Sigma}$ not be an identity matrix when the undifferenced disturbances are i.i.d., but since the mapping from $\boldsymbol{\Omega}$ to $\boldsymbol{\Sigma}$ is many to one, it is possible to say very little about $\boldsymbol{\Omega}$ from knowledge of $\boldsymbol{\Sigma}$ unless additional assumptions are made.
} 
where $\phi_{p-1}$ represents the $p-1$-variate normal probability density function, and

$$
A_{j}=\left\{\begin{array}{lr}
\left\{\mathbf{z}_{i}^{*}: z_{i j}^{*}>0, z_{i j}^{*}>z_{i,-j}^{*}\right\} & \text { for all } j \leq p-1 \\
\left\{\mathbf{z}_{i}^{*}: z_{i 1}^{*}<0, z_{i 2}^{*}<0, \ldots, z_{i, p-1}<0\right\} & \text { for } j=p
\end{array}\right.
$$

The sampling density is then given by

$$
f\left(\mathbf{y} \mid \boldsymbol{\beta}, \mathbf{\Sigma}^{*}\right)=\prod_{i=1}^{n} \prod_{j=1}^{p} \operatorname{Pr}\left(y_{i j}=1 \mid \boldsymbol{\beta}, \mathbf{\Sigma}^{*}\right)^{y_{i j}} .
$$

The posterior density of $\boldsymbol{\beta}$ and $\boldsymbol{\Sigma}^{*}$ is given by Bayes theorem as

$$
\pi\left(\boldsymbol{\beta}, \mathbf{\Sigma}^{*} \mid \mathbf{y}\right) \propto f\left(\mathbf{y} \mid \boldsymbol{\beta}, \mathbf{\Sigma}^{*}\right) \pi(\boldsymbol{\beta}) \pi\left(\mathbf{\Sigma}^{*}\right)
$$

where, $\pi(\boldsymbol{\beta})$ and $\pi\left(\boldsymbol{\Sigma}^{*}\right)$ denote the (independent) prior densities of $\boldsymbol{\beta}$ and $\boldsymbol{\Sigma}^{*}$, respectively.

To estimate this model, we iteratively sample from this posterior distribution. The key to avoiding many of the computational difficulties of estimating the MNP using frequentist or Bayesian methods is data augmentation (Albert and Chib 1993). Even though the actual value of $\mathbf{z}^{*}$ is unobserved, we know how it is distributed conditional on the data and other model parameters. By including draws of these unobserved values of $\mathbf{z}_{i}^{*}$ inside what would otherwise be a Markov chain Monte Carlo (MCMC) sampling scheme for a slightly reformulated seemingly unrelated regression (SUR) model, we are able to fit the MNP model at minimal computational cost. We employ the Chib, Greenberg, and Chen (1998) MCMC sampling algorithm.

\subsection{The MNL Model}

The MNL model assumes that the disturbances in Equation 1 are independently and identically distributed according to the Weibull distribution (McFadden 1974). The choice probabilities then take the following form,

$$
\operatorname{Pr}\left(y_{i j}=1 \mid \gamma, \delta\right)=\frac{\exp \left(\mathbf{v}_{i j}^{\prime} \gamma+\mathbf{w}_{i j}^{\prime} \delta\right)}{\sum_{k=1}^{p} \exp \left(\mathbf{v}_{i k}^{\prime} \gamma+\mathbf{w}_{i k}^{\prime} \delta\right)}
$$

where, for reasons of identification, the $p$ th (baseline) row of $\mathbf{W}_{i}$ has been set equal to zeros, and accordingly every $p$ th column of $\mathbf{W}_{i}$ has also been 
deleted. Although the posterior density of this model is not available in closed form, a sequence of draws from it can be constructed using the Metropolis-Hastings algorithm (Chib, Greenberg, and Chen 1998).

\subsection{Bayesian Model Comparison}

Central to the idea of Bayesian model comparison and hypothesis testing is the Bayes factor, which provides a convenient means to assess the amount of evidence in favor of one scientific theory operationalized as a statistical model versus that for another (Kass and Raftery 1995, 777). The Bayes factor, then, allows us to compare competing MNP and MNL models of the same data, on the scale of probability. Unlike frequentist hypothesis tests (such as the likelihood ratio test), the Bayes factor is not interpreted with respect to critical values.

Applying Bayes theorem, Kass and Raftery (1995) demonstrate that the posterior odds in favor of model $j$ relative to model $k$ are given by

$$
\frac{\operatorname{Pr}\left(M_{j} \mid y\right)}{\operatorname{Pr}\left(M_{k} \mid y\right)}=\frac{\operatorname{Pr}\left(y \mid M_{j}\right)}{\operatorname{Pr}\left(y \mid M_{k}\right)} \frac{\operatorname{Pr}\left(M_{j}\right)}{\operatorname{Pr}\left(M_{k}\right)} .
$$

The first term on the right-hand side is known as the Bayes factor, and the second term consists of the prior probabilities of the two models (hypotheses). Thus, the Bayes factor $B_{j k}$ between models $M_{j}$ and $M_{k}$ is equal to the posterior odds ratio of the models in question when uniform priors are assumed. More formally, the Bayes factor is given by

$$
B_{j, k}=\frac{\operatorname{Pr}\left(y \mid M_{j}\right)}{\operatorname{Pr}\left(y \mid M_{k}\right)}=\frac{\int_{\theta} f\left(y \mid \theta, M_{j}\right) \pi\left(\theta \mid M_{j}\right) d \theta}{\int_{\theta} f\left(y \mid \theta, M_{k}\right) \pi\left(\theta \mid M_{k}\right) d \theta} .
$$

Note that $\int_{\theta} f(y \mid \theta, M) \pi(\theta \mid M) d \theta$ is the normalizing constant of the posterior density of $\theta$ conditional on $y$ and model $M$. This normalizing constant is known as the marginal likelihood. Inspection of the above integral reveals that the marginal likelihood is the expected value of the sampling density given our prior beliefs about the model parameters $\theta$. In other words, the marginal likelihood is proportional to the probability of seeing the data that actually were observed before they were observed. That the value of the marginal likelihood depends on the priors assumed suggests two things. First, priors need to be chosen carefully so that they are reasonably accurate reflections of the researcher's beliefs. Second, the Bayes factor favors parsimonious models over more complex competitors. This is the case unless the 
researcher's beliefs about the parameters from the more complex model are more certain and in line with the observed data, or the likelihood under the complex model is much more sharply spiked in the high prior density region of the parameter space. We employ the method of Chib (1995) to calculate the marginal likelihood.

\section{The Data}

Our data come from two sources: Euro-Barometer 11 (Rabier and Inglehardt 1981) and the European Political Parties' Middle-Level Elites (EPPMLE) data set (ISEIUM 1983). Our dependent variable is the response to a question on the Euro-Barometer: "If there were a general election tomorrow and you had a vote, which party would you support?"4 Since 1977, four major parties have captured at least 135 of the 150 seats in the Dutch parliament, with numerous special interest parties splitting the remainder. These four parties are the Labor Party (PvdA), Democrats 1966 (D'66), the Christian Democratic Appeal (CDA), and the Liberals (VVD). This dependent variable is unordered and polychotomous.

We use the EPPMLE study, a survey of delegates to the European party conferences in 1979, to operationalize issue positions. Our elite and mass surveys have similar issue questions, conducted at nearly the same time. Spatial theories assume that a voter's preferences can be represented by a point in a well-defined issue space and that party policy can be represented by points in this same space. Unfortunately, neither these issue locations nor relevant information about the issue space itself (e.g., number of dimensions) is directly observable.

Yet, we do have measures of voter preferences on specific issues such as abortion availability, governmental control of industry, and the need to reduce economic inequality. These specific responses are presumably a result of the voters' more general political preferences (the unobservable spatial locations) and a random error. Thus, we can use factor analysis to recover estimates of the latent issue positions. Further, since we hypothesize that voters evaluate parties in terms of their general political preferences, we employ the same scoring coefficients used to project voter issue responses into the more general issue space to project parties into this same space. ${ }^{5}$

${ }^{4}$ Question 262. Elections were held in the Netherlands in 1977 and 1981. We use the Euro-Barometer 11 data set because it is the only Euro-Barometer survey with issue questions that match the EPPMLE survey.

${ }^{5}$ There are alternative strategies one could use to measure party positions. The most common approach, using party manifestos to compute the relative positions of the parties (Budge, Robertson, and Hearl 1987), is problematic because it does not locate voters and parties in the same issue space. We need voter and party positions in a common space to define a distance metric. Asking individuals in a mass survey to place each party on an issue scale (e.g., Alvarez and Nagler 1998) allows analysis of most European cases, but is problematic because it requires that dimensionality of the 
To obtain bliss points for each voter and party, we start by performing factor analysis on seven questions from the Euro-Barometer $11 .^{6}$ These questions address: public control of private enterprise, abortion, terrorism, nuclear energy, multinational corporations, environmental protection, and income redistribution. Based on the exploratory factor analysis (EFA) results, it appears that two factors are present, contrary to assertions of Van der Eijk and Niemöller (1987). We then estimate a two-factor confirmatory factor analysis (CFA) model, where the factors are forced to be uncorrelated. Our final model fits the data quite well: the ratio of the $\chi^{2}$ statistic to the degrees of freedom for the model is low (below two); the model root mean square residual is low (below 0.05); and the CFI is high (above 0.90). We call our first factor a general economic (left-right) factor; the second, a measure of preferences over the scope of government. Scoring coefficients for the components of the factors are obtained and used to construct bliss points for the individual voters. ${ }^{\text {? }}$

For the corresponding question in the EPPMLE data set, we rescale the question to match the coding scheme used above. Each of the EPPMLE questions includes a category of no opinion, coded as a five-point scale (strongly favor, favor, no opinion, oppose, strongly oppose). The EuroBarometer data set includes no such category. Thus, we recode responses to the EPPMLE questions on a 1 to 4 scale with no opinion coded as 2.5. This will over-weight the elite positions towards the middle of the issue space, but we are confident that our measures of party position are reliable because

issue space be assumed a priori and requires the assumption that each individual places each party in an identical space. Further, because it seems likely that voters may perceive the location of their favored party as closer to them than is objectively warranted, using such a measure of distance would bias results in favor of the spatial model. Finally, the accuracy of the implied measurement model cannot be gauged in a statistical sense. The advantage of using a party elite survey is that one can gauge the issue positions of the elites and thus make inferences about the party positions relative to voter positions.

The "curvilinear disparity" (May 1973) is a potential problem with our approach. That is, since we use the preferences of "middle-level elites" to obtain measures of party positions, our measures might be biased towards extremism. On this score, we note two points. First, our "middle-level elites" are members of national party conferences. In May's terminology, such elites are actually of the same strata as government executives, legislators, and other high-level elites. Second, in the Netherlands (a country with a very pure form of proportional representation), the underlying theoretical reasons to expect representatives to be more centrist than middle-level elites are absent. Consequently, the advantages that our method offers over other methods of obtaining comparable elite and mass issue positions outweigh the potential problems stemming from curvilinear disparity.

${ }^{6}$ The issue question wordings for both surveys and tables of factor loadings are available at http://psweb.sbs.ohio-state.edu/ajps/.

${ }^{7}$ The CFA was performed via maximum, likelihood in SAS using PROC CALIS. A productmoment (Pearson) correlation matrix was used as input. Reanalysis of this data in LISREL 8.20 using Browne's asymptotically distribution free method and a polychoric correlation matrix as input produces nearly identical factor loadings and scoring coefficients. 
we use the median on each dimension to place the party, which is a very robust measure of central tendency (with a breakdown point of 50\%). After standardizing the EPPMLE responses, we use regression scoring to obtain bliss points for individual elites. For each of the four parties, we take the median of all elites on both of the issue dimensions as our measure of party position. We then compute the squared spatial distance between the voter and each party and include them in our spatial models as a choice-specific covariate.

To assess the explanatory power of social-structural theory, we include a battery of demographic characteristics as independent variables. Three variables capture notions of class and religion: Manual Labor (coded 1 if the respondent is a manual laborer and 0 otherwise), Religion (scored 0 for those indicating religion is not important and up to 4 for those indicating religion is very important), and Income ( 0 for those with income less than 750 Guilders; up to 12 for those with income greater than 3751 or more Guilders). We also include other commonly-used demographic characteristics: Town Size captures urban/rural splits in the electorate by measuring subjective town size (with 1 indicating small town/rural, 2 indicating middle-size town, and 3 indicating city), and Education (the age the respondent finished formal education, ranging from 1 indicating 14 years or younger-to 9, indicating 22 years or older). In addition, we include the age of each of the parties as a choice-specific covariate. ${ }^{8}$

\section{RESUlts}

We specify three models of voter choice in the Netherlands: a spatial, a social-structural, and a joint spatial/social-structural. For each set of covariates, we estimate an MNP and an MNL. ${ }^{9}$ For each model, we compute

${ }^{8}$ The inclusion of at least one choice-specific covariate is sufficient to avoid what Keane (1992) has termed "fragile identification" in the multinomial probit model. Without at least one choicespecific covariate, MNP estimates are sensitive using either classical or Bayesian techniques. We include the age of each of the parties as a proxy for the organizational strength of each of the four parties under study. Other measures are problematic. Party membership of each of the four parties is a possibility. Yet the number of citizens belonging to parties has historically been low, with a fair amount of volatility (Andeweg and Irwin 1993, 63-65). Since the size of a party's war chest is, to some extent, determined by the party's age (Andeweg and Irwin 1993, 67), we have adopted the party's age as a proxy for organizational strength. The age of the CDA was calculated as the average age of each of the three parties (ARP, CDU, KVP) which merged to form the CDA.

${ }^{9}$ For all of the models, we use the following proper prior on $\boldsymbol{\beta}: \boldsymbol{\beta} \sim \mathcal{N}_{k}(\overline{\boldsymbol{\beta}}, \mathbf{A})$, where $\overline{\boldsymbol{\beta}}$ is a $k \times 1$ vector of means. $\overline{\boldsymbol{\beta}}$ takes the value .5 if our theory indicates that the coefficient should be positive, -.5 if our theory indicates that the coefficient should be negative, and 0 for constants. The prior variance on $\boldsymbol{\beta}$ is $\mathbf{A}=3 * \mathbf{I}_{\mathbf{k}}$. The prior on the free main-diagonal elements of $\mathbf{\Sigma}^{*}$ is $\boldsymbol{\Sigma}_{j j}^{*} \sim \mathcal{N}(1,3)$. The prior on the off-diagonal elements $\boldsymbol{\Sigma}^{*}$ is $\boldsymbol{\Sigma}_{i j}^{*} \sim \mathcal{N}(.5,3)$. These mean values correspond to an undifferenced error covariance matrix with mean $\mathbf{I}_{\mathbf{p}}$. After performing extensive robustness checks, it is clear that our results do not substantively differ under different prior means and variances. 
the marginal likelihood from which we compute Bayes factors. Due to space constraints, we only summarize the posterior density for the joint spatial/ social-structural model. Table 1 presents the results from the joint MNP and MNL. First, the spatial distance differs significantly from zero (with a 95 percent BCI falling below 0) in both models in the hypothesized direction. As expected, status as a manual laborer exerts a positive effect (with a 95 percent $\mathrm{BCI}$ falling above 0 ) on the probability of voting for the PvdA; increasing educational levels have a negative effect on this probability. Being a manual laborer increases the likelihood of voting for the CDA relative to D'66, as does religious importance. Increasing educational levels have a negative effect on voting for the CDA. So, too, do increases in personal income tend to increase the probability of voting for the VVD. The only substantive difference between these models is that, under the MNL specification, living in an urban area increases one's chances of voting for CDA. This effect is not significant for the MNP. Overall, these results shows that the Dutch voters are not merely slaves to their social-structural pillars, nor are they purely issue-minded utility maximizers. Social structure matters, even when controlling for issue preferences. Futhermore, even after controlling for meaningful demographic factors, Dutch voters cast ballots for parties closest to them in an issue space.

Now we turn to the Bayes factors. These results appear in Table 2. Among our MNP specifications, the spatial model is better supported by the data than the social-structural model. This is not surprising because the spatial model provides a parsimonious, predictively accurate explanation of the vote choice. For the MNP, the joint model does best of all, which indicates some remnants of pillarisation remain in the Netherlands. Among the MNL specifications, we find the same results. The joint model fits better than the spatial, but the spatial continues to out-perform the social-structural model. Thus, even when controlling for placements in the social pillars, Dutch voters cast ballots for parties that espouse similar issue views.

The more interesting results come when comparing MNP and MNL specifications. Here the joint MNP beats all other models. This implies that the MNP is more likely to have produced our sample data than the MNL model. Thus, although for these data the substantive conclusions may not differ, it would be inappropriate to apply MNL to study Dutch voting behavior given our specification. In other contexts, using the incorrect model could lead to incorrect inferences. We conclude that the spatial model is the best individual explanation of Dutch voting behavior and that our joint MNP specification provides the best overall understanding of voting behavior in the Netherlands.

To investigate the substantive importance of our estimates, we compute predicted probabilities of voting (using Equation 2) for the four parties when 
Table 1. Summary of MNP and MNL Posterior Densities from the Dutch Joint Spatial/Social-Structural Model

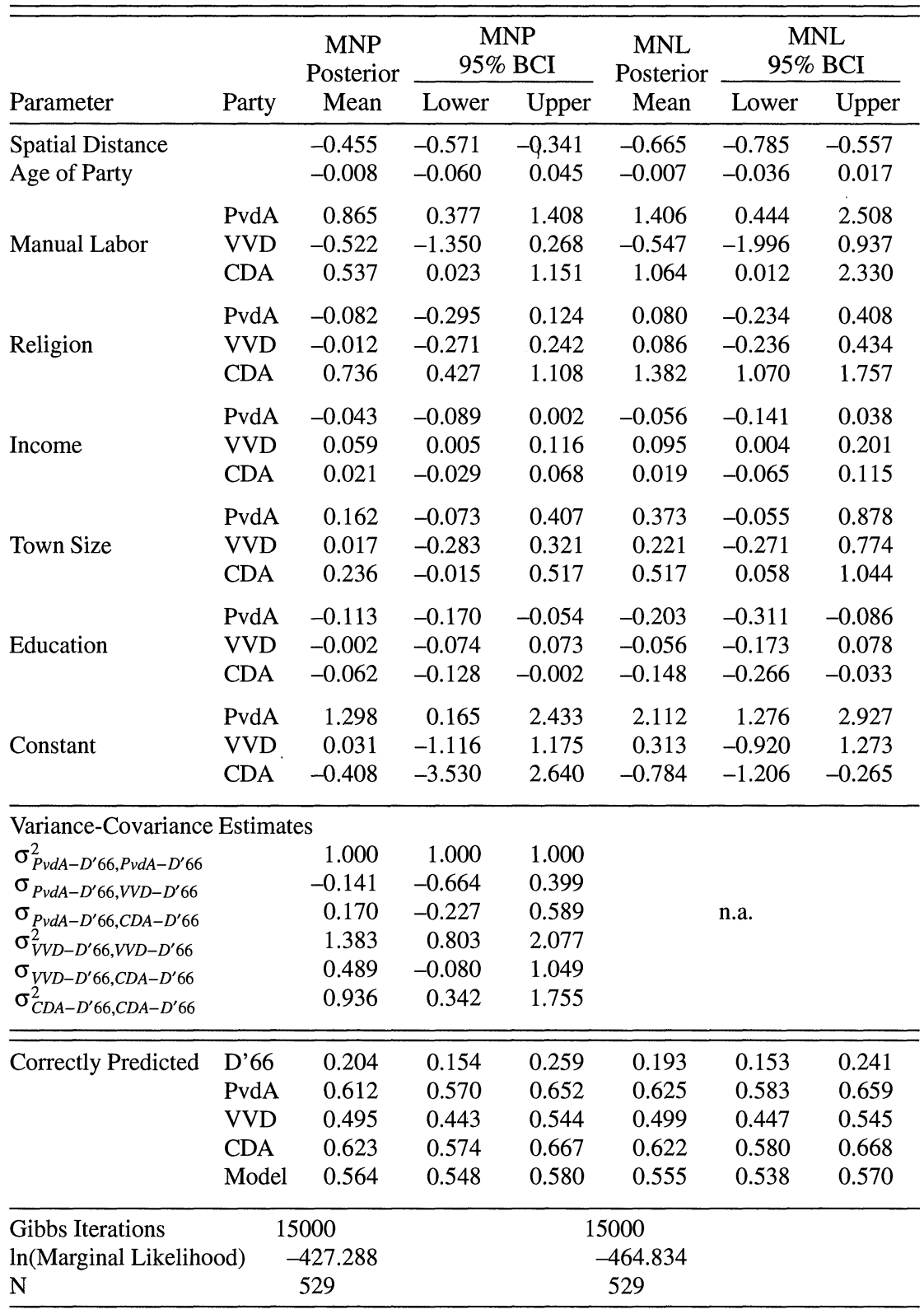

Note: The abbreviation 95\% BCI represents the Bayesian Credible Interval. This interval summarizes the central $95 \%$ of the posterior density. 
Table 2. $L o g$ Bayes Factors $\left[\ln \left(B_{j k}\right)\right]$ for Model $j$ vis-à-vis Model $k$ of Dutch Voting Behavior

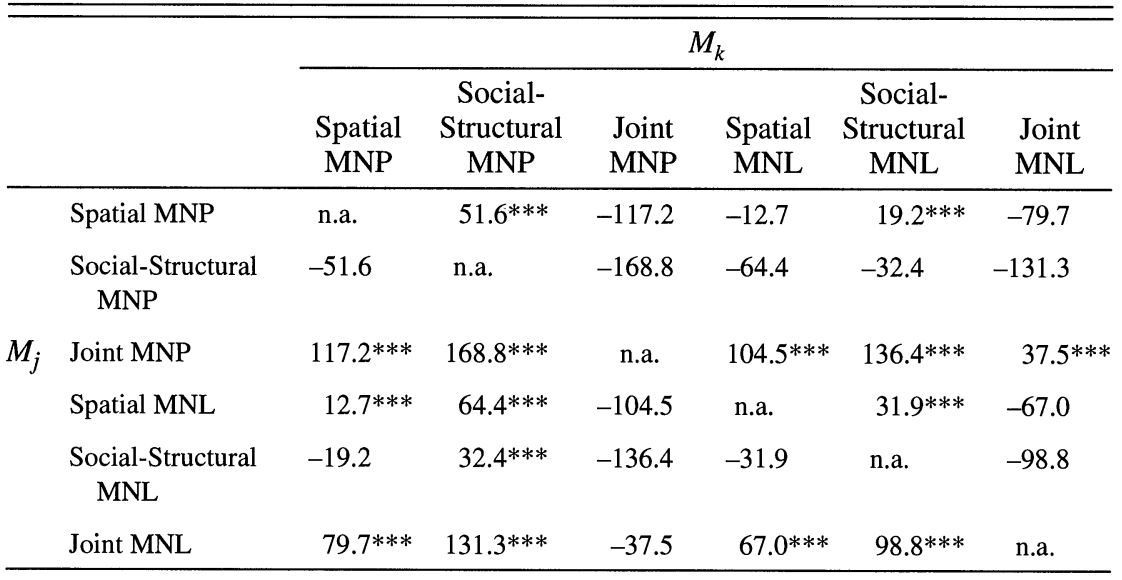

*** = Very Strong Support for $M_{j},{ }^{* *}=$ Strong Support for $M_{j},{ }^{*}=$ Positive Support for $M_{j}$.

changing two important variables: Manual Labor and Religion. To get a sense of the relative magnitudes of these effects, we fix the other explanatory variables at their means and calculate vote probabilities over the ranges of these two variables. Since the variables are discrete, the resulting vote probabilities can be presented in a table. Table 3 presents the probability of voting for each of the four parties as occupational status and religious importance are allowed to vary. Several points are immediately apparent. As we would expect, occupational status exerts the strongest effect on the probabilities of voting for PvdA and VVD. All else being equal, manual laborers are 1.64 times more likely to vote for PvdA, and 23 percent less likely to vote for VVD than are other voters. Compared to the effect of religious importance, the effect of occupational status is relatively small. Religious importance, by contrast, generally exerts a large effect on the probabilities of voting for the four parties under study. As expected, the largest effect is on the probability of voting for CDA. Those who feel that religion is very important are 16.3 times more likely to vote for CDA than are those for whom religion is unimportant. The effect of religiosity on the probabilities of voting for the other parties is of a lesser magnitude, but not trivial.

\section{Extension: Great Britain}

To put our results on the Netherlands in perspective, we consider data from another multi-party democracy, Great Britain (see also Alvarez and 


\section{Table 3. Impact of Changes in Main Social-Structural Variables on Dutch Voting Behavior}

\begin{tabular}{lcccc}
\hline \hline Variable & $\operatorname{Pr}(\operatorname{PvdA})$ & $\operatorname{Pr}\left(\mathrm{D}^{\prime} 66\right)$ & $\operatorname{Pr}(\mathrm{CDA})$ & $\operatorname{Pr}(\mathrm{VVD})$ \\
\hline Manual Labor $=0$ & 0.307 & 0.175 & 0.326 & 0.193 \\
Manual Labor $=1$ & 0.504 & 0.053 & 0.398 & 0.045 \\
Religion $=0$ & 0.483 & 0.218 & 0.058 & 0.241 \\
Religion $=1$ & 0.391 & 0.181 & 0.238 & 0.190 \\
Religion $=2$ & 0.249 & 0.010 & 0.543 & 0.109 \\
Religion $=3$ & 0.113 & 0.033 & 0.812 & 0.042 \\
Religion $=4$ & 0.036 & 0.007 & 0.946 & 0.011 \\
\hline
\end{tabular}

Note: All other variables have been set at their mean values.

Nagler 1998; Whitten and Palmer 1996). ${ }^{10}$ Similar to the Netherlands, Great Britain prior to the 1970s featured a system of electoral allegiances that were closely related to social class. During the mid-to-late 1970s, however, class lines began to soften, and voting behavior became more volatile, more influenced by electoral campaigns, and more issue-oriented. It is also interesting to note that the campaign leading up to the 1979 election focused almost exclusively on the major party leaders and was in this sense more "presidential" than other British electoral campaigns (Butler and Kavanagh 1980, 322).

We operationalize the spatial and the social-structural explanations in the same fashion as in the Netherlands. Here, too, we find two dimensions, a general economic (left-right) factor and preferences over the scope of government. ${ }^{11}$

We present results from the joint model in Table 4. These results are quite strong, as the squared spatial distance parameters are significantly different from zero and in the hypothesized direction for both models. For both our MNP and MNL models, manual laborers are significantly more likely to vote for Labour, and the religious are more likely to vote for the Conservatives. Additionally, in both specifications those with higher incomes are significantly more likely to vote for the Conservatives. Again, these results indicate that even when controlling for social-structural considerations, there

${ }^{10} \mathrm{An}$ election was held in Great Britain in 1979. In this election, the Conservatives displaced the ruling Labour government that had been in place since 1974. To avoid fragile identification, we include the outcome in the October 1974 election as a choice-specific variable.

${ }^{11}$ The factor analysis results and posterior density summaries for all models are available at http://psweb.sbs.ohio-state.edu/ajps/. Here, however, we use Browne's asymptotically distributionfree method and a polychoric correlation matrix and estimate the factor model in LISREL 8.20, allowing the factors to be correlated. 
Table 4. Summary of MNP and MNL Posterior Densities from
the UK Joint Spatial/Social-Structural Model

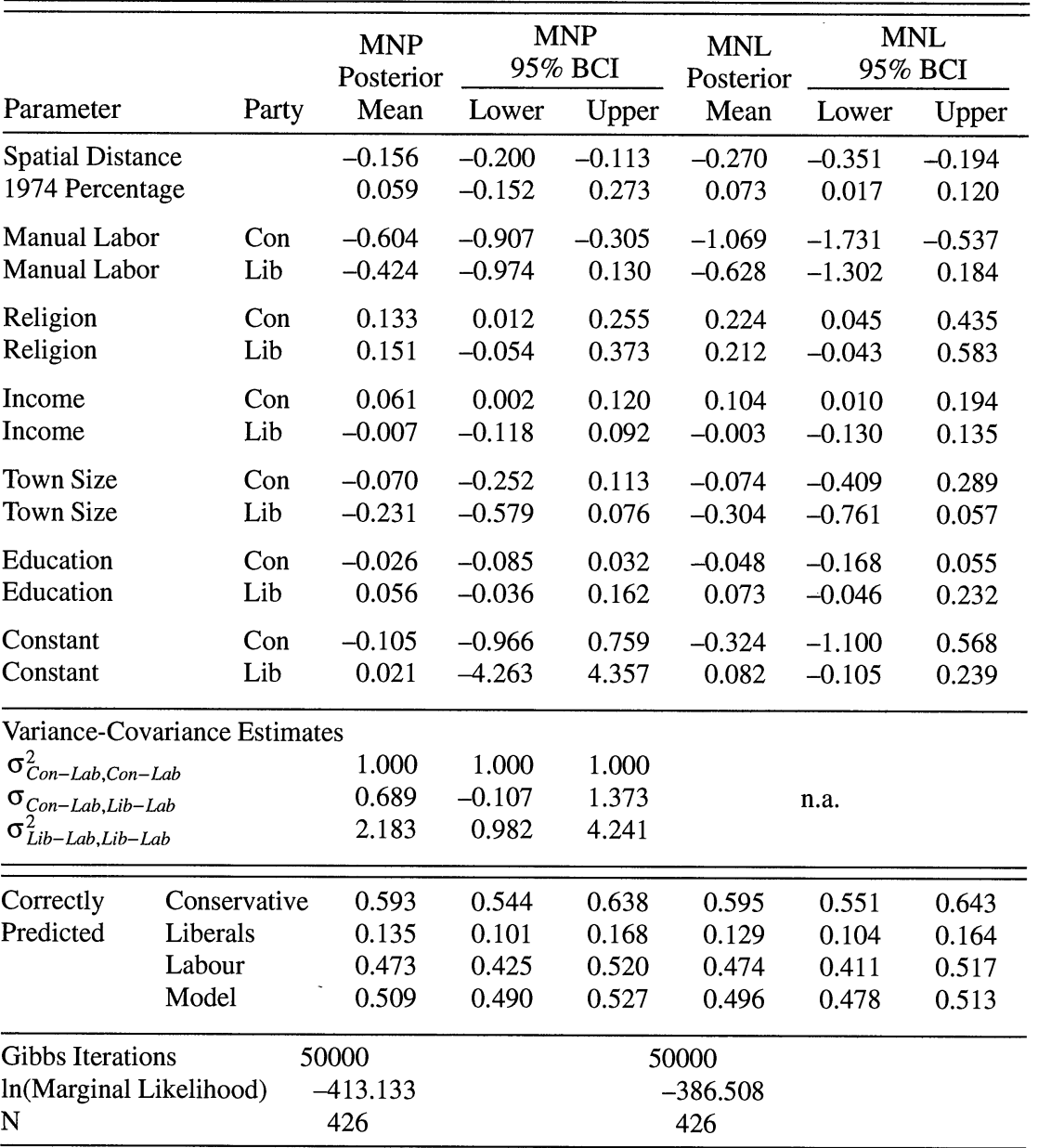

Note: The abbreviation 95\% BCI represents the Bayesian Credible Interval. This interval summarizes the central $95 \%$ of the posterior density.

is evidence of issue voting in the United Kingdom. Finally, in the MNL specification, the October 1974 vote percentage has a significant, positive effect on current vote choice. This effect does not appear in the MNP results.

To test the explanatory power of the models, we present Bayes factors in Table 5. Among the MNPs, the spatial model is the best explanatory model. 
Table 5. Log Bayes Factors $\left[\ln \left(B_{j k}\right)\right]$ for Model $j$ vis-à-vis Model $\boldsymbol{k}$ of UK Voting Behavior

\begin{tabular}{|c|c|c|c|c|c|c|c|}
\hline & & \multicolumn{6}{|c|}{$M_{k}$} \\
\hline & & $\begin{array}{l}\text { Spatial } \\
\text { MNP }\end{array}$ & $\begin{array}{l}\text { Social- } \\
\text { Structural } \\
\text { MNP }\end{array}$ & $\begin{array}{l}\text { Joint } \\
\text { MNP }\end{array}$ & $\begin{array}{l}\text { Spatial } \\
\text { MNL }\end{array}$ & $\begin{array}{l}\text { Social- } \\
\text { Structural } \\
\text { MNL }\end{array}$ & $\begin{array}{l}\text { Joint } \\
\text { MNL }\end{array}$ \\
\hline & Spatial MNP & n.a. & $41.0 * * *$ & $19.7 * * *$ & -9.2 & $18.5^{* * *}$ & -7.0 \\
\hline & $\begin{array}{l}\text { Social-Structural } \\
\text { MNP }\end{array}$ & -41.0 & n.a. & -21.4 & -50.3 & -22.5 & -48.0 \\
\hline \multirow[t]{4}{*}{$M_{j}$} & Joint MNP & -19.7 & $21.4^{* * *}$ & n.a. & -28.9 & -1.2 & -26.6 \\
\hline & Spatial MNL & $9.2 * * *$ & $50.3 * * *$ & $28.9 * * *$ & n.a. & $27.8 * * *$ & $2.3 *$ \\
\hline & $\begin{array}{l}\text { Social-Structural } \\
\text { MNL }\end{array}$ & -18.5 & $22.5 * * *$ & $1.2 *$ & -27.8 & n.a. & -25.5 \\
\hline & Joint MNL & $7.0 * * *$ & $48.0^{* * *}$ & $26.6^{* * *}$ & -2.3 & $25.5^{* * *}$ & n.a. \\
\hline
\end{tabular}

This implies that one only needs to know the issue preferences of a British voter to explain their voting behavior. Similarly, for the MNL, the spatial model is the best explanatory model. Although it is a close call between the spatial and joint MNLs, there is positive support for the spatial MNL as the best explanatory model.

When comparing across theories and models, the spatial MNL outperforms all models. Thus, contrary to our findings from the Netherlands, one only needs to know issue positions to explain voting behavior in Britain. This fits with accounts of the 1979 British election as an election in which the previously strong class-lines in British politics began to break down in favor of other, election-specific determinants of voter choice (Butler and Kavanagh 1980; Rose and McAllister 1986). Additionally, our MNL specification does best, implying that given these specifications, the computationally easy MNL does a sufficient job.

\section{Discussion}

Our substantive results indicate that the spatial theory of voting is the best individual explanatory model of Dutch voting behavior. This result runs counter to much of the existing literature on the Netherlands. However, the spatial explanation is not the whole story, as a joint spatial social-structural model is the best overall model given our specification and data. Dutch voters do not simply vote based on their place in society; nor do they strictly vote based on party issue positions. This result can interpreted as evidence 
of the slow erosion of the Dutch social pillars or, in a more positive light, as the permanence of social cleavages. Nonetheless, the spatial distance between a voter and a party exerts a very strong effect on voter choice in the Netherlands, even when controlling for other factors such as religious belief and social class.

In Great Britain, by contrast, the spatial model beats not only a sociological model, but also a joint model containing both sociological variables and a measure of ideological distance. This result comports with the conventional wisdom that the 1979 British general election was a contest in which previously strong class lines were blurred, and party policy positions became increasingly important.

The ability to compare MNP and MNL specifications on a scale of probability using the Bayes factor is the methodological contribution of this paper. By adopting a Bayesian approach, we are able jointly to test competing explanations and models of voter choice. Our results demonstrate that in the Dutch case, the MNP is the appropriate model to employ given our choice of covariates. Our substantive conclusions would not have been greatly affected by choosing the wrong model. However, the Bayes factor between the joint MNP and the joint MNL suggests that the distributional assumptions underlying MNL are incorrect given our specification and data. In Britain, the MNL model does better than the MNP given our operationalization. Our British example highlights the importance of assessing the fit of various specifications as opposed to functional forms. One should not take the results in this article and conclude that there is no difference between results reached from MNP and MNL. Clearly that is not the case. Alvarez and Nagler (1998) show how one can make incorrect inferences about competing explanations by assuming a particular functional form. Our results here show that although one would have picked the correct explanation, important substantive coefficients would be misinterpreted had the wrong model been assumed.

Manuscript submitted September 3, 1997.

Final manuscript received March 24, 1999.

\section{REFERENCES}

Albert, James H., and Siddhartha Chib. 1993. "Bayesian Analysis of Binary and Polychotomous Response Data." Journal of the American Statistical Association 88:669-679.

Alvarez, R. Michael, and Jonathan Nagler. 1998. "When Politics and Models Collide: Estimating Models of Multi-Candidate Elections." American Journal of Political Science 42:55-96.

Andeweg, Rudy B., and Galen A. Irwin. 1993. Dutch Government and Politics. New York: St. Martin's Press. 
Budge, Ian, David Robertson, and David Hearl. 1987. Ideology, Strategy, and Party Change. Cambridge: Cambridge University Press.

Butler, David, and Dennis Kavanagh. 1980. The British General Election of 1979. London: MacMillan.

Chib, Siddhartha. 1995. "Marginal Likelihood From the Gibbs Output." Journal of the American Statistical Association 90:1313-1321.

Chib, Siddhartha, Edward Greenberg, and Yuxin Chen. 1998. "MCMC Methods for Fitting and Comparing Multinomial Response Models." Typescript. Washington University in St. Louis.

DeGroot, Morris H. 1986. Probability and Statistics. Reading, Mass.: Addison Wesley.

Downs, Anthony. 1957. An Economic Theory of Democracy. New York: Harper \& Row.

Dutter, Lee E. 1985. "Spatial Preferences and Voter Choices in the Dutch Electorate."Comparative Political Studies 18:251-263.

Van der Eijk, Cees, and Kees Niemöller. 1987. "Electoral Alignments in the Netherlands." Electoral Studies 6:17-39.

Enelow, James, and Melvin Hinich. 1984. The Spatial Theory of Voting: An Introduction. Cambridge: Cambridge University Press.

Institut für Sozialwissenschaften and Europa-Institut of the Universität Mannheim. 1983. European Elections Study: European Political Parties'Middle-Level Elites. Köln: Köln Zentralarchiv.

Kass, Robert E., and Adrian E. Raftery. 1995. "Bayes Factors." Journal of the American Statistical Association 90:773-795.

Keane, Michael P. 1992. "A Note on Identification in the Multinomial Probit Model." Journal of Business \& Economic Statistics 10:193-200.

Lacy, Dean, and Barry C. Burden. 1999. "The Vote-stealing and Turnout Effects of Ross Perot in the 1992 US Presidential Election.” American Journal of Political Science 43:233-255.

Lijphart, Arend. 1975. The Politics of Accomodation: Pluralism and Democracy in the Netherlands, 2nd ed. Berekely: University of California Press.

May, John D. 1973. "Opionion Structure of Political Parties: The Special Law of the Curvilinear Disparity." Political Studies XXI:135-151.

McFadden, Daniel. 1974. "Conditional Logit Analysis of Qualitative Choice Behavior." In Frontiers in Econometrics, ed. Paul Zarembka, New York: Academic Press.

Rabier, Jacques-Rene, and Ronald Inglehardt. 1981. Euro-Barometer 11 - April, 1979: The Year of the Child in Europe. Ann Arbor, Mich.: Inter-University Consortium for Political and Social Research.

Rose, Richard, and Ian McAllister. 1986. Voters Begin to Choose: From Closed-Class to Open Elections in Britain. London: Sage Publications.

Schofield, Norman J., Andrew D. Martin, Kevin M. Quinn, and Andrew B. Whitford. 1998. "Multiparty Electoral Competition in the Netherlands and Germany: A Model Based on Multinomial Probit." Public Choice 97:257-293.

Whitten, Guy D., and Harvey D. Palmer. 1996. "Heightening Comparativists' Concern for Model Choice: Voting Behavior in Great Britain and the Netherlands." American Journal of Political Science 40:231-260. 